\title{
Twitter en la campaña electoral de 2012
}

\author{
Eva Salgado Andrade
}

$\mathrm{E}$ ste trabajo explora el uso de Twitter en el marco de la campaña electoral por la presidencia de México en 2012 a partir de dos preguntas: ¿quién tuiteaba y para quiénes lo hacía?, ¿qué se tuiteaba? ${ }^{1}$ Se pretende contribuir a la reflexión sobre nuevas formas de acción social y de producción de la opinión pública vinculadas con las redes sociales virtuales, entre las que destaca la emergencia del Movimiento \#YoSoy132. Vista en conjunto, la efervescencia del tema electoral en Twitter, algunos de cuyos trending topics determinaron la agenda mediática de la contienda política, revela el interés de una población, mayoritariamente joven, dispuesta a explotar el potencial, aún insuficiente pero progresivo, de las redes sociales para hacer escuchar sus demandas por una verdadera democracia.

${ }^{1}$ Es inevitable que en este trabajo usemos neologismos perfectamente comprensibles como "tuitear" o "tuitero", que de hecho quedarán incorporados en la xxiII Edición del Diccionario de la Real Academia Española, lo que por otra parte no hace sino reflejar cómo el lenguaje ha debido adaptarse a nuevas prácticas sociales.

\section{INTRODUCCIÓN}

¿Qué sucedió en Twitter durante las elecciones para la presidencia en México en 2012? Este trabajo presenta los resultados de una exploración discursiva, semiológica, lingüística, sociológica y antropológica en torno a la convergencia de dos fenómenos igualmente relevantes. En primer lugar, el advenimiento de la tecnología para crear formas de información años. En segundo lugar, con la campaña electoral por la presidencia como telón de fondo, la sociedad mexicana fue testigo del nacimiento de nuevas formas de acción social, protagonizadas por una población, jóvenes universitarios en su mayoría, dispuesta a hacer escuchar sus demandas y denuncias ante las irregularidades del proceso, como la inequidad de las televisoras privadas con los contendientes, el oscuro manejo de las encuestas, la insatisfacción ante la manipulación mediática en torno a algunos episodios de la campaña, como la visita de Enrique Peña Nieto a la Universidad Iberoamericana (Ibero), suceso que, alimentado por

\section{Twitter in the Electoral Campaign of 2012}

Eva Salgado Andrade: Centro de Investigaciones y Estudios Superiores en Antropología Social-Distrito Federal, México esalgado@ciesas.edu.mx

Desacatos, núm. 42, mayo-agosto 2013, pp. 217-232 
la respuesta social colectiva, daría lugar a la creación del Movimiento \#YoSoy132. El trabajo inicia con la breve reconstrucción de la reciente y vertiginosa historia de Twitter, microblog que comenzó con el modesto propósito de mantener comunicación entre personas cercanas, y la forma en que fue rediseñado por sus usuarios. Después se exponen los resultados de esta exploración en dos vertientes: quién tuiteaba y para quién lo hacía, y qué se tuiteaba. En las conclusiones reflexionamos sobre las posibles causas que impidieron que la efervescencia de temas relacionados con las elecciones en las redes sociales se tradujeran en un avance democrático real.

\section{TWITTER Y SU VERTIGINOSA HISTORIA}

Twitter es un servicio de creación de microblogs, desarrollado en marzo de 2006 por Jack Dorsey, en San Francisco, California. Su nombre proviene de la palabra inglesa "tweet": trinar, gorjear, parlotear. redes go-gulf.com, en mayo de 2012 Twitter contaba con 555 millones de usuarios en el mundo: 57\% mujeres y $43 \%$ varones. El rango de edad predominante era de 26 a 34 años - 30\% - y de 35 a 44 años $-27 \%$ - A partir de su creación, esta red social virtual ha tenido una evolución insospechada, incluso para sus creadores y por supuesto para quienes han acometido su estudio. Hace apenas cuatro años, en una entrevista con el diario El País (Reventós, 2008), Jack Dorsey definía así su invento:

Un servicio que te conecta con tu gente, que responde a preguntas de lo que estás haciendo en ese momento mediante el teléfono móvil, la web, la mensajería instantánea y a través del correo electrónico. Te permite estar en contacto con tu gente en tiempo real.

En la misma entrevista, ante una provocadora pregunta de su entrevistador, resultaba evidente que el propio Dorsey no vislumbraba la potencialidad de su invento:

Pregunta: Hay gente que considera que a nadie le interesa saber cuando va usted al baño...

Respuesta: Siempre se puede mirar desde esa perspectiva, pero si habla con mi madre le dirá que está en un error. Yo soy de Sant Louis y llevo más de 12 años fuera de casa. Con Twitter estamos en contacto diario, sabemos qué estamos haciendo uno y otro sin necesidad de hablar por teléfono. Los microblogs van dirigidos a tu audiencia particular, que pueden ser dos únicas personas, pero las más importantes para ti. Mandarles esos mensajes no es ninguna banalidad. Sin duda es un servicio menos creativo que el del blog, pero no pretende ir dirigido a grandes audiencias.

Un año después, Twitter no pasaba de ser concebido como una herramienta para que los usuarios hicieran pública su vida privada, sobre todo entre sus allegados. Grossman opinaba así sobre su funcionalidad:

Un servicio en línea que puede usarse para mandar notas — de 140 caracteres o menos - al mundo a través de la red, mensajería instantánea y SMs. Lo usan para comentar lo último que están haciendo, comiendo, viendo, sintiendo, etc., a su familia, amigos y cualquiera que los esté siguiendo (Grossman, citado en Yus, 2010: 158).

Conviene aclarar la distinción entre público y privado. Böckelmann (1983: 52) sostiene que lo público no debe entenderse necesariamente como opuesto a lo privado, sino que se refiere a los temas, intenciones, estructuras y oportunidades que son "generales", es decir, que gozan de omnipresencia. La esfera de lo público, continúa el autor, se integra por especialistas — que argumentan públicamentey por consumidores - que asimilan lo público- . Lo privado no necesariamente excluye lo público, pues aquél está mediatizado por la acción de los medios de comunicación social. Rosa Jiménez Cano reflexionaba sobre la mutación experimentada por Twitter, para pasar del ámbito de lo privado a lo público, en un reportaje publicado a propósito de su quinto aniversario: 
Twitter quería ser un sistema de envío de mensajes a un grupo de amigos. Los que hoy entendemos como "followers" - seguidores-. De hecho, la pregunta inicial era “¿Qué estás haciendo?", hace un año se cambió a “¿Qué está pasando?”. Esta mutación se puede interpretar como un cambio hacia el exterior, como la constatación de que Twitter dejaba de ser un medio minimalista, interpersonal, para pasar a dirigirse a un lector indeterminado al otro lado del ordenador o el móvil (Jiménez, 2011).

Paulatinamente, Twitter, al igual que otras redes sociales, mostró su capacidad para dotar "a la gente de una manera nueva y convincente de socializar y mantenerse en contacto" (Carr, 2011: 196). Yus (2010: 159) ofrece una forma de entender la mutación que hizo posible que un medio concebido para la comunicación íntima con amigos y familiares se convirtiera virtualmente en el esparcidor instantáneo, mundial y simultáneo de informaciones y opiniones al advertir que con frecuencia las nuevas tecnologías de la información y de la comunicación son rediseñadas por sus usuarios según patrones no previstos por los creadores de dichas tecnologías. ¿Qué ocurrió en Twitter en relación con el proceso electoral de 2012? ¿Qué formas de expresión social y acción colectiva se valieron de los rediseños de esta nueva tecnología? ¿Tuvieron alguna repercusión en el desarrollo del episodio electoral? Para intentar las primeras respuestas es preciso referirnos, así sea someramente, a algunos componentes del panorama electoral reciente: luego de 12 años de gobierno panista, la sociedad experimenta serios rezagos en materia económica, social, política, cultural y científica. En apenas seis años otro flagelo se ha instaurado de manera alarmante en nuestro país: la guerra o estrategia contra el narcotráfico o crimen

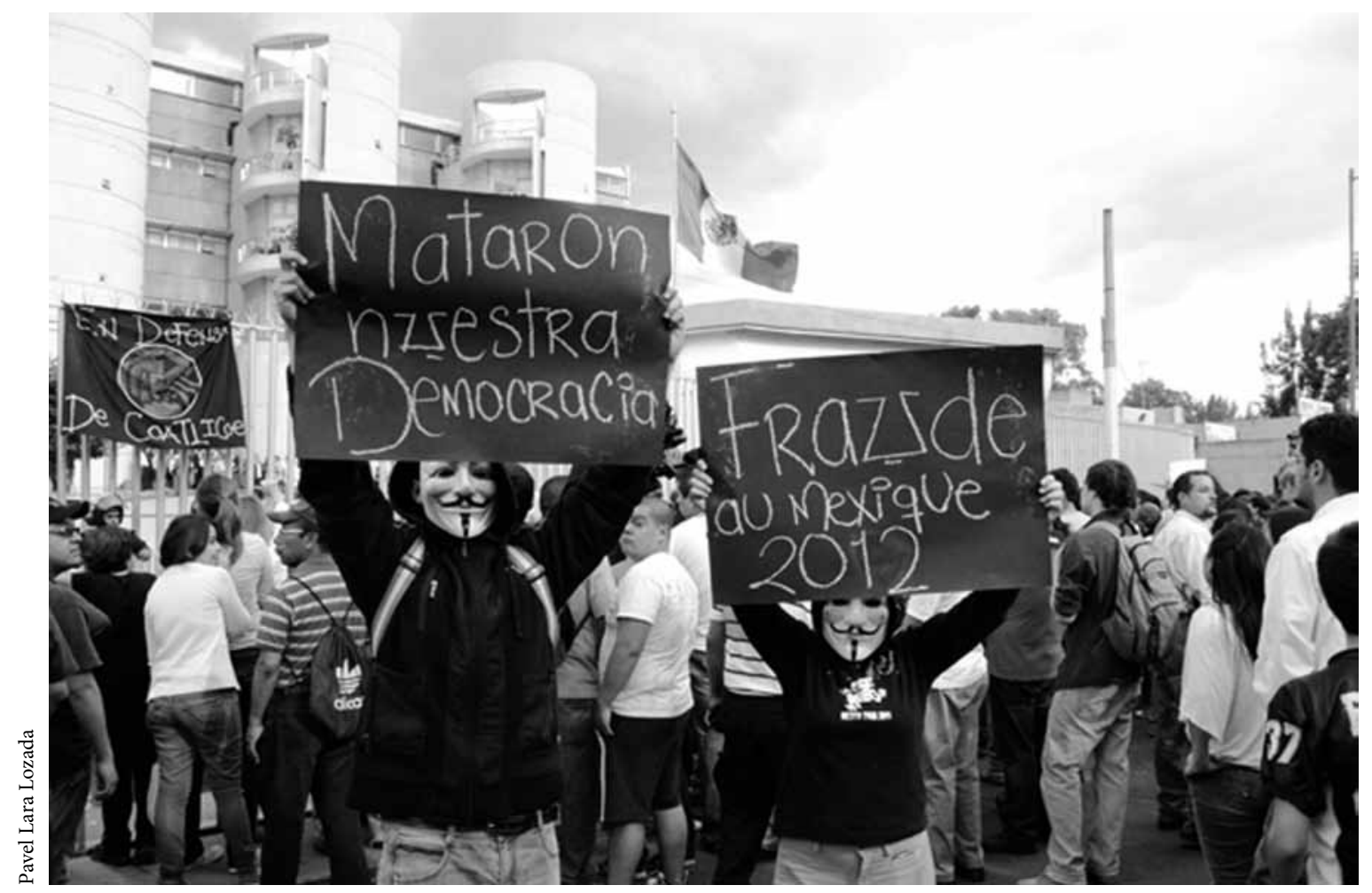

Frente a las instalaciones del Tribunal Federal Electoral (Trife), manifestantes protestan contra la entrega de la constancia de mayoría a Enrique Peña Nieto. 
organizado -la denominación es irrelevante, en tanto las consecuencias no se modifican- se ha traducido en una cifra que supera ya 80000 muertos, 240000 desplazados y cerca de 15000 desaparecidos. Para amplios sectores de la sociedad, este proceso electoral representaba la oportunidad para un cambio, y no sólo de partido. Flotaban aún en el ambiente las heridas derivadas de las elecciones de 2006, que Aziz define como "conflictivo y polarizante, que pareció hacer que México regresara a los tiempos de desconfianza, del discurso del fraude y de la protesta callejera" (Aziz, 2007: 14).

Asimismo, en 2012 el monopolio de la información por parte de las cadenas de televisión se tornaba cada vez más evidente, como lo era también la preferencia hacia el entonces candidato, y a la postre presidente electo, Enrique Peña Nieto (EPN), postulado por la alianza del Partido Revolucionario Institucional (PRI) y el Verde Ecologista de México (PVEM). Los elevados gastos de campaña del PRI constituyeron uno de los temas fuertemente impug-

nados, que pese a ser denunciados y documentados de manera constante - por ejemplo, mediante el monitoreo y difusión frecuentes de anuncios espectaculares al por mayor en buena parte de las carreteras y avenidas principales del país - no fueron sancionados por el Instituto Federal Electoral (IFE). Frente a este panorama, en el ambiente público electoral la democracia real se avizoraba como extremadamente vulnerable. Esto conformó un clima de insatisfacción creciente entre una parte de la ciudadanía, de por sí interesada en la participación política, así como la súbita aparición del movimiento social \#YoSoy132, integrado principalmente por jóvenes universitarios, cuyo origen estaba muy ligado con Twitter, como se advierte en el hecho de la denominación que eligieron.

Cabe destacar que si bien la relevancia de Twitter se explica en parte por las cifras, que nos remiten a un desmedido crecimiento potencial a nivel mundial, para su comprensión también es necesario evaluar aspectos de tipo cualitativo. Islas (2012) proporciona interesantes cifras en relación con la situación de Twitter en México durante el periodo explorado. En marzo de 2012 se calculaba un total de 4103200 cuentas, de las cuales 2480000 , es decir, $60.44 \%$, estaban activas. Estos números, aunque relevantes, no deberían tomarse con demasiado optimismo, al menos no de manera cuantitativa. De acuerdo con Islas, el porcentaje total de tuiteros mexicanos, tomando en cuenta la población total según datos censales, apenas representa 3.65\%, y si se consideran sólo los usuarios activos el porcentaje desciende a $2.20 \%$. Sin embargo, este modesto $2.20 \%$ - o una proporción incluso menor si partimos de la base de que no todos los tuiteros estaban ocupados con las elecciones- logró incidir en la agenda mediática política en 2012.

¿Cómo sistematizar algunas reflexiones en torno a lo que los candidatos, los electores, los seguidores, los detractores, los críticos, los apáticos, los militantes furibundos, los navegadores ocasionales, los curiosos, etcétera, encontraron en los últimos meses buscando en Twitter razones para votar o no votar? ¿Qué imágenes, sentimientos, valoraciones o representaciones identificaron en los candidatos por los que sentían simpatía o antipatía, o incluso por los que parecían generarles indiferencia? ¿Cómo puede sintetizarse, si ello es viable, la andanada de nuevos paradigmas, tipologías, códigos y hasta nuevos lenguajes que surgieron en un universo virtual tan insólito y mutable como Twitter?

Para no perdernos en elucubraciones proponemos como punto de partida recurrir a modelos teóricos ampliamente comprobados, como la propuesta de Roman Jakobson (1981: 352-360) respecto del proceso de la comunicación. Haremos un recorrido exploratorio siguiendo como hilo conductor los inveterados elementos del proceso de la comunicación, que implican la existencia de un emisor que dirige su mensaje a un destinatario, a través de un canal que determina sus propios códigos, en función de un contexto específico, es decir, en su carácter de discurso. Para ello, dividimos la exploración en dos apartados: quién tuiteaba y para quiénes lo hacía, y qué se tuiteaba. Este énfasis en quiénes y qué es también 
retomado por Gee (2002: 13), quien advierte que un enunciado sólo tiene sentido si comunica un quién y un qué. Por un quién se pretende expresar una identidad socialmente situada. El qué expresa una actividad socialmente situada. Los quiénes son múltiples y no necesariamente personas, incluso pueden ser "anónimos" y aun así tener pleno sentido. Los quiénes y los qués no son unidades separadas: quiénes somos forma parte de qué hacemos, y lo que hacemos se reconoce en parte por quién lo hace. Para quien desee incursionar en este océano de discursos virtuales es conveniente retomar algunas de las sugerencias metodológicas que Kozinets (2010: 89) formula en su obra Netnography - término derivado de la combinación de net y etnography-: buscar para el análisis comunidades en línea que sean 1) relevantes, es decir, relacionadas con el tema y preguntas de investigación; 2) activas, con comunicaciones regulares; 3 ) interactivas, al tener un flujo de comunicación entre los participantes, y 4) heterogéneas, al convocar a participantes diversos, entre otras cualidades.

\section{¿QUIÉN TUITEABA Y PARA QUIÉN LO HACÍA?}

A diferencia de lo que por lo regular ocurre en los medios de comunicación masiva, las redes sociales virtuales dan, al menos potencialmente, las mismas posibilidades a cualquier persona para que abra una cuenta y sea seguida, es decir, puede convertirse en un emisor y saber con exactitud cuántas personas han decidido expresamente ser sus destinatarios. Al dar de alta una cuenta el usuario es invitado a seguir ciertos pasos, no todos obligatorios, por cierto. Puede registrar su propio nombre y elegir además un nombre de usuario de menos de 15 caracteres - que usará para identificarse en las respuestas y menciones en Twitter-, añadir su fotografía e información de su perfil, que proporciona al sistema ciertas preferencias. En Twitter los emisores pueden ser en principio aquellos tradicionalmente identificados como líderes de opinión en la esfera pública, como periodistas, columnistas, académicos o los propios medios de comunicación que han debido cubrir todo flanco posible de interacción con sus usuarios. Además, un creciente número de actores sociales, de manera individual o colectiva, emplean este espacio como un foro novedoso para difundir sus actividades, sus opiniones, sus emociones, en torno a prácticamente cualquier tema. En el contexto electoral que nos ocupa, es evidente que los contendientes encontraron en Twitter un recurso adicional para mantenerse en contacto con los electores o con los detractores. Twitter proveyó el formato; los candidatos, el perfil. La imagen seleccionada debía ser más que una ocurrencia aleatoria. Tratándose de candidatos inmersos en el contexto electoral, había obvias razones para suponer qué se quería decir por medio de la imagen. Como advierte Knapp (1982: 134), es posible desarrollar reglas de expresión personales basadas en las necesidades o exigencias de la profesión, por ejemplo, los políticos. ¿Qué decían sobre sí mismos los candidatos en su perfil de Twitter?

En su perfil de candidato por el Movimiento Progresista, Andrés Manuel López Obrador (AMLO), @lopezobrador_, se autodenominó sólo con sus nombres de pila, quizá para dar una sensación de afabilidad y confianza hacia su electorado. De estilo más bien lacónico, no se ofrecía más información que "Sitio oficial" y la dirección electrónica de su página web. La única imagen era una fotografía del candidato, sobre un fondo gris, en un encuadre medium close up, que daba relevancia al rostro, con los ojos entrecerrados y la comisura de los labios hacia arriba, lo que permitía ver la dentadura, en una expresión que podría leerse como una mezcla de confianza y felicidad. Para el perfil de Enrique Peña Nieto, @EPN, se eligió también mostrar en primer plano el rostro de candidato de la coalición PRI-PVEM. Esta imagen se obtuvo del encuadre de una fotografía de toma abierta, completada con otra de fondo, que mostraba al candidato en un acto de campaña electoral, rodeado de personas aparentemente de condición 


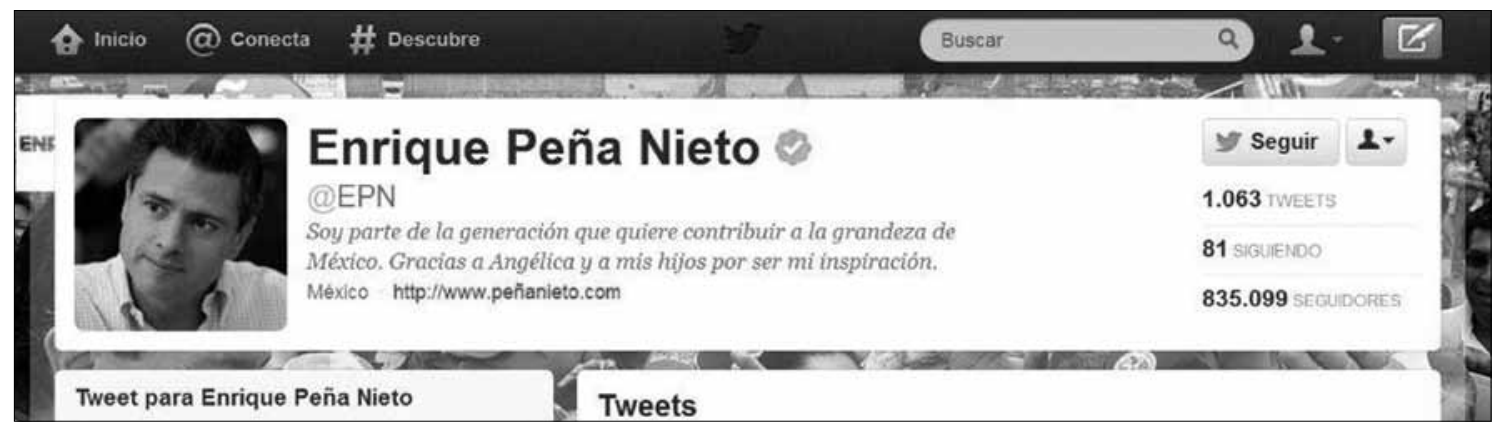

Perfil de Twitter de Enrique Peña Nieto.

económica baja. La expresión del rostro dejaba ver apenas una leve extensión de la comisura de los labios cerrados, los ojos muy abiertos no se dirigían hacia el frente, parecían observar algo a la distancia. En el perfil, el candidato fue prolijo en ejercicios de autoconstrucción de hablante: "Soy parte de la generación que quiere contribuir a la grandeza de México. Gracias a Angélica y a mis hijos por ser mi inspiración". Con estas referencias sobre su persona parecía nio con la actriz Angélica Rivera en noviembre de 2010, ampliamente publicitado en televisión abierta.

Para la candidata por el partido en el gobierno, Josefina Vázquez Mota (JVM), en @JosefinaVM, se eligió una imagen distinta a la de los demás candidatos. En su fotografía aparecía en primer plano, aunque no centrada, su mano izquierda en alto, mostrando un enorme tache azul en toda la palma, que simbolizaba el mensaje enviado hacia sus simpatizantes para que votaran por ella. Un encuadre de otra fotografía la mostraba acompañada de miembros de su equipo de campaña. Como fondo de esta construcción visual se distinguía un mitin electoral, en el que destacaba el rostro fervoroso de una joven en pleno grito de lucha. En la información que ofrecía sobre sí misma, además de dar testimonio de su obvio carácter de candidata, se ponía énfasis en la función fática, es decir, centrada en los canales de comunicación que se abrían con las redes sociales: "Candidata del PAN a la Presidencia de México. Sigue a @Equipo_JvM para información en tiempo real.

Envíame tus propuestas y comentarios a: josefina@ josefina.mx". En este pequeño conjunto verbal destaca la construcción de interlocutores mediante el uso de la forma coloquial "tú" — "Sigue", "Envíame" -, así como la recurrencia excesiva al nombre de pila en el diseño de sus nombres de usuario: JosefinaVM y josefina@josefina.mx. Por último, Gabriel Quadri, cuya candidatura no tuvo mayor relevancia, si acaso tratar de convencer a los electores indecisos, en @g_quadri presentaba una fotografía en medium shot, en una postura de aparente informalidad - recordemos la incongruente insistencia de Quadri por deslindarse de los políticos durante el primer debate organizado por el IFE-, con la mirada de frente hacia la cámara, sobre un fondo blanco. La descripción era un minicurrículum que informaba el perfil profesional y de intereses del candidato por Nueva Alianza: "Ingeniero Civil, Economista, Ambientalista y deportista. Candidato a la Presidencia de la República Mexicana por Nueva Alianza”.

Además de los datos que ofrecían los perfiles de los candidatos, en el formato desarrollado por Twitter otra información susceptible de lecturas y análisis podía hallarse en el número de tweets enviados, las personas a quienes seguían y la cantidad de seguidores que tenían, tema que también fue objeto de polémicas en la red, debido al incremento en la cantidad de usuarios que no parecían seguir un patrón normal de conducta en Twitter, y parecían más bien seguidores maquilados en aras de dar la impresión de amplia aceptación en redes sociales. Uno de 


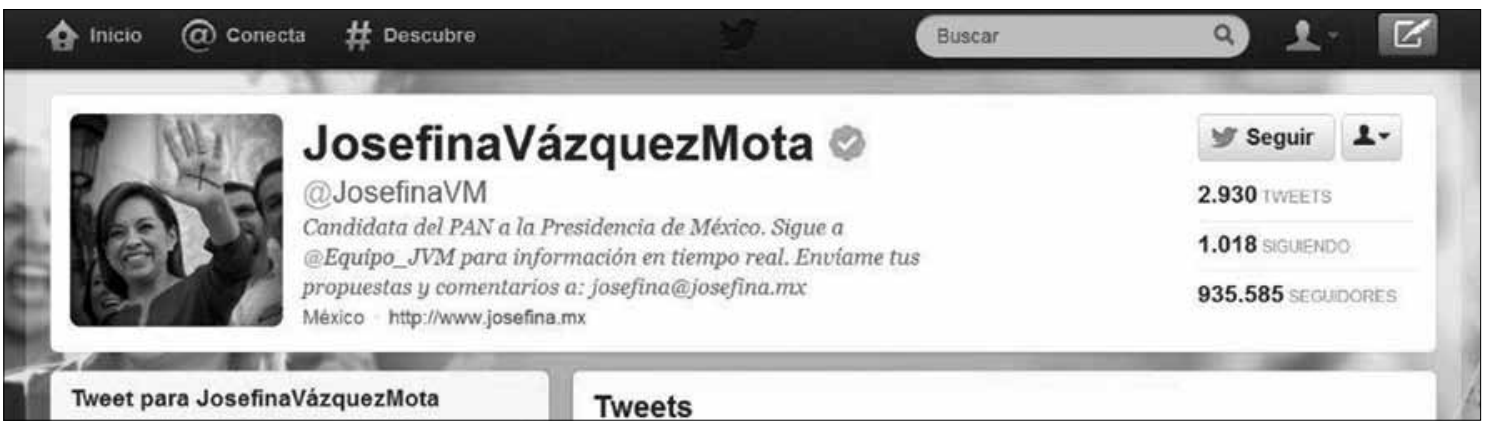

Perfil de Twitter de Josefina Vázquez Mota.

los ejemplos más sonados fue el de los llamados "peñabots", tuiteros contratados por el equipo de campaña de EPN. Para dar una idea del universo virtual al que nos referimos, presentamos las cifras del número de seguidores de los cuatro candidatos el 17 de junio de 2012: AMLO: 737617; EPN: 839719; JVM: 932 775; Gabriel Quadri: 214 161. También es interesante observar el registro del número de usuarios seguidos por los candidatos: AMLO: 785; EPN: 81; JVM: 1018, y Quadri: 210. Llama la atención la reducida cantidad de usuarios a los que siguieron los candidatos, en particular EPN, cuya proporción respecto de los cientos de miles que aparentemente lo seguían fue de $0.009 \%$. Ofrecemos la información de un monitoreo similar realizado meses después de la contienda, el 27 de octubre de 2012. Sin ahondar más por el momento en la información que revelan las cifras, podemos ver que la actividad en Twitter continuó: el número de seguidores de AMLO y de EPN incrementó, en tanto las cifras para JVM disminuyeron drásticamente -AMLO: 970355 seguidores; EPN: 1158264 seguidores; JVM: 791218 seguidores; Quadri: 216226 seguidores-. En conjunto, el total de tweets enviados por los candidatos, según cifras obtenidas en octubre de 2012, fue muy similar entre tres de ellos, en tanto que la candidata emitió casi el doble: AMLO: 1778 tweets; EPN: 1466 tweets; JVM: 3215 tweets; Quadri: 1854 tweets.

Estas cifras eran un termómetro de la opinión pública en los grados de aceptación o rechazo de los candidatos. De allí la práctica, varias veces denunciada, de fabricar seguidores. Bourdieu (1984: 222-223) plantea que el capital específico de un dirigente es tan sólo un valor fiduciario que depende de la representación, de la opinión, de la creencia, de la fidelidad; el hombre político, al igual que el hombre de honor, es un individuo especialmente vulnerable a las suposiciones, a las calumnias, al escándalo y a todo lo que amenace la creencia y la confianza. En suma, "el hombre político es el que dice: 'Dios está de nuestra parte. El equivalente de 'Dios está de nuestra parte' es hoy en día 'la opinión pública está de nuestra parte"' (Bourdieu, 1984). Añadiríamos, siguiendo el tema que nos ocupa: "las redes sociales están de nuestra parte". En este proceso de búsqueda de legitimidades y consensos por parte de la opinión pública se entiende por qué los partidos buscan "la confianza del individuo, valiéndose más de personas que de programas y disposiciones, que pudieran parecer extraños, impenetrables y abstractos al ciudadano" (Böckelmann, 1983: 35). Lo anterior explica cómo este alud de voces pudo ser objeto de algún tipo de conducción u orquestación por parte de los equipos electorales en contienda. Además de los candidatos, un creciente número de emisores se interesó en participar en la vida electoral. Más allá de los tradicionalmente reconocidos como opinadores profesionales, hubo una oleada de informaciones y juicios, expresiones de fobias y filias, dudas y certezas, angustias y alegrías, miedos y denuncias, emociones y convicciones, que exhortaban a votar o no por alguno de los contendientes. Vale la pena hacer 


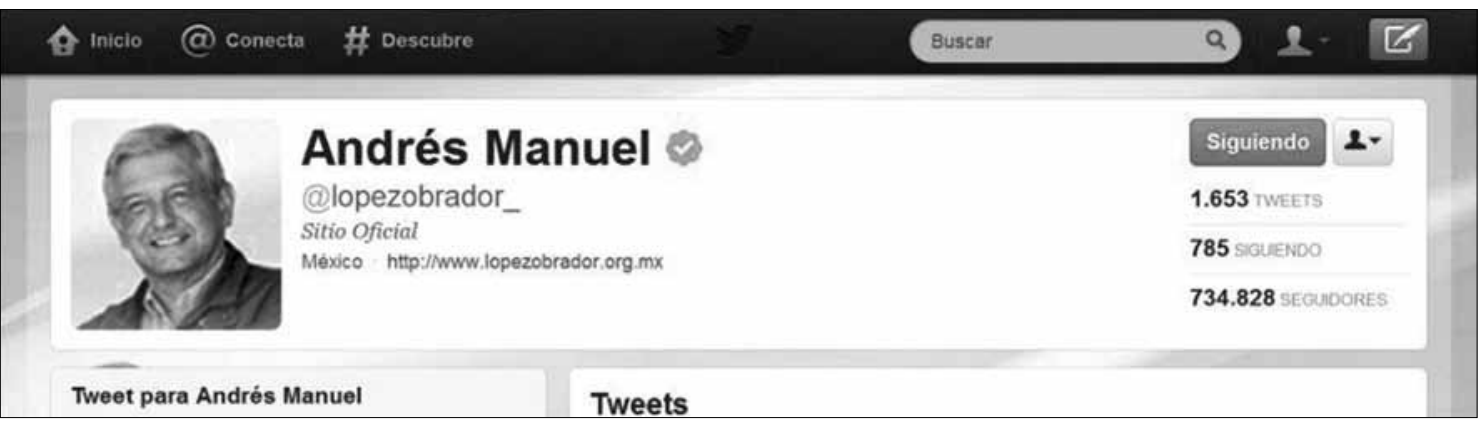

Perfil de Twitter de Andrés Manuel López Obrador.

notar que esta red social permitió manifestarse no sólo a emisores individuales, también dio lugar a uno de los procesos más interesantes de la contienda: la emergencia de comunidades o grupos de opinión que encontraron una forma de organización inusitadamente veloz y eficaz. Destaca la comunidad de jóvenes universitarios autodenominada \#YoSoy132, que sin dudas ganó un lugar en la historia política del país, cuya indignación latente frente a la cerrade la información pudo encontrar en Twitter una resonancia excepcional.

En un brevísimo recuento, conviene recordar que el punto de partida fue la visita de Enrique Peña Nieto a la Ibero el 11 de mayo de 2012. Cuando el candidato, al referirse a su gestión como gobernador del Estado de México, aceptó y casi se vanaglorió de haber ordenado en 2006 mano dura para contener a los manifestantes en San Salvador Atenco - hecho que ha pasado a la historia por las múltiples agresiones a derechos humanos de manifestantes y observadores - fue impugnado con severidad por la comunidad universitaria. El episodio de las imprecaciones a Peña Nieto y su salida entre gritos de “ $¡ F u e r a$, fuera!” se difundió con gran velocidad en las redes sociales y surgieron trending topics - de los que hablaremos más adelante- no sólo en México, sino en el mundo, como \#UIA, \#EPNenlauia o el \#MeEscondoEnElBañoComoepn, este último en alusión a versiones que difundieron que supuestamente el candidato había terminado

atrapado en los baños de la universidad. Pedro Joaquín Coldwell, presidente del PRI, declaró que las agresiones habían provenido de un grupo de intolerantes ajenos a la universidad, seguramente enviados por perredistas o lopezobradoristas, lo que enardeció aun más los ánimos de la comunidad estudiantil. La información difundida al día siguiente, en particular la nota de primera plana "Éxito de Peña en la Ibero, pese a intento orquestado de boicot" en los diarios de la Organización Editorial Mexicana, dirigida por el empresario Mario Vázquez Raña, representó una prueba contundente de la parcialidad con que se daban a conocer temas relacionados con la campaña. En las redes circuló profusamente una fotografía que mostraba a Peña Nieto y a Vázquez Raña con el expresidente Carlos Salinas de Gortari.

El equipo de campaña del PRI produjo un video en el que participaron jóvenes del Instituto Tecnológico Autónomo de México (ITAM) para reconstruir una versión amable de la visita del candidato a la Ibero. Transmitido en Youtube, este video registró en unas cuantas horas un alud de "dislikes", es decir, la valoración negativa de los visitantes. ${ }^{2}$ Una versión en fragmentos de este video puede encontrarse en $<$ http://www.youtube.com/watch? $\mathrm{v}=\mathrm{qcKn} 6 \mathrm{Aq}$ PnUs $>$. Como reacción a este manejo tendencioso de

\footnotetext{
${ }^{2}$ Según las pautas establecidas por esta red virtual, los materiales pueden calificarse con un "me gusta" - "like" - o "no me gusta" - “dislike"-.
} 


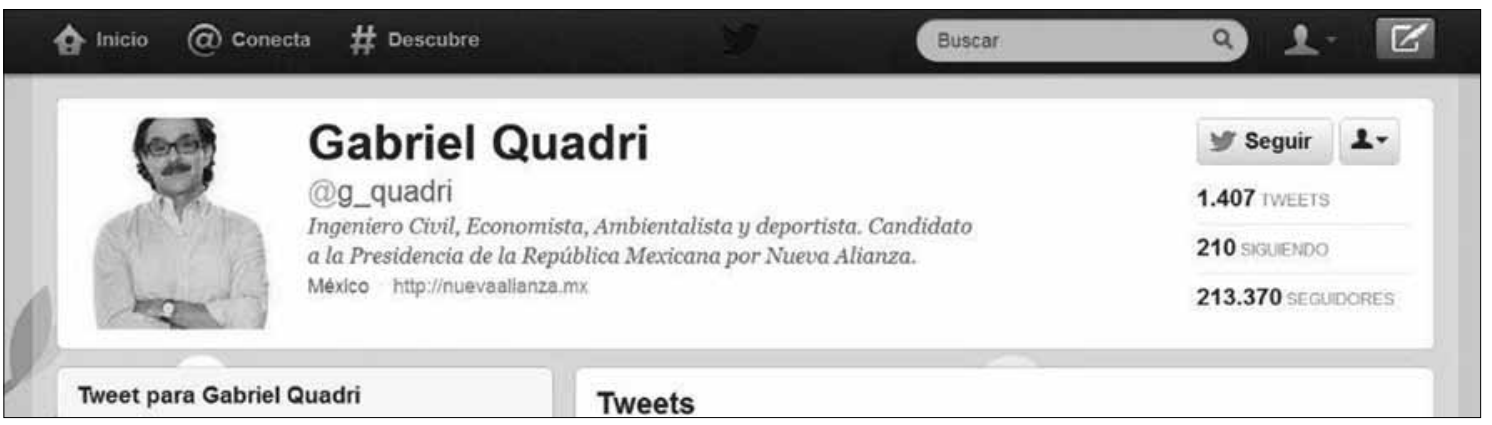

Perfil de Twitter de Gabriel Quadri.

la información, 131 estudiantes de la Ibero difundieron un video, muy visitado en redes sociales, en el que se identificaban como alumnos regulares de la universidad para desmentir las versiones que sugerían que se habría tratado de un grupo de provocadores ajenos a dicha casa de estudios. A partir de la respuesta de los usuarios de redes sociales a este video surgió el trending topic \#SomosMasDe131, para manifestar el respaldo y la adhesión a la causa de los estudiantes, que demandaban una información más plural y equitativa, entre otras exigencias. En cuestión de días, se creó el Movimiento \#YoSoy132, integrado esencialmente por jóvenes universitarios inscritos en instituciones tanto públicas como privadas de todo el país. Con los meses, y no exento de altibajos, el Movimiento se ha erigido como representante de la juventud mexicana cada vez más inconforme, no sólo respecto de la vulnerable democracia, sino también en relación con otros asuntos de interés público.

Como ocurre en general en toda la web, es posible que los usuarios no sean una persona o comunidad identificable en el mundo real. El propio sistema advierte qué se permite o qué se prohíbe. Por ejemplo, las "parodias" están autorizadas, es decir, hacer burla o imitación de algo en tono de broma. Se permite a los usuarios de Twitter crear cuentas paródicas, comentarios y cuentas de fans. El sistema despliega otras instrucciones para que un usuario aclare que su cuenta es una parodia:
Nombre de usuario: El nombre de usuario no debe ser exactamente el mismo del sujeto de la parodia, imitación o la celebridad; para hacer esto más claro, debes distinguir la cuenta con un calificativo tal como "no soy", "falso" o "fan".

Nombre real: El nombre en el perfil no debe listar exactamente el nombre del sujeto sin alguna palabra distintiva, tal como "no soy", "falso" o "fan" (Centro de Ayuda de Twitter).

Por el contrario, la "suplantación" está expresamente prohibida por el sistema, que la define como "pretender ser alguien que no eres en internet. La suplantación de la identidad pretende engañar a otros y es prohibido bajo las Reglas de Twitter" ("El glosario de Twitter", Centro de Ayuda de Twitter). Es muy común que los usuarios asuman una identidad falsa y se hagan pasar, con distintos propósitos, por personajes públicos: políticos, artistas, deportistas, periodistas, etc. En el contexto electoral que nos ocupa, la suplantación de identidad estuvo a la orden del día. Bajo el nombre de "Andrés Manuel López Obrador", "Enrique Peña Nieto" o "Gabriel Quadri” decenas de usuarios aprovecharon Twitter para apoyar o denostar a los candidatos. A pesar de lo delicado que, en apariencia, puede resultar el fenómeno de no saber con certeza quién es el verdadero destinador o emisor, conviene recordar que esto forma parte de los propios códigos de funcionamiento de la red. Hine propone una reflexión interesante al respecto: 


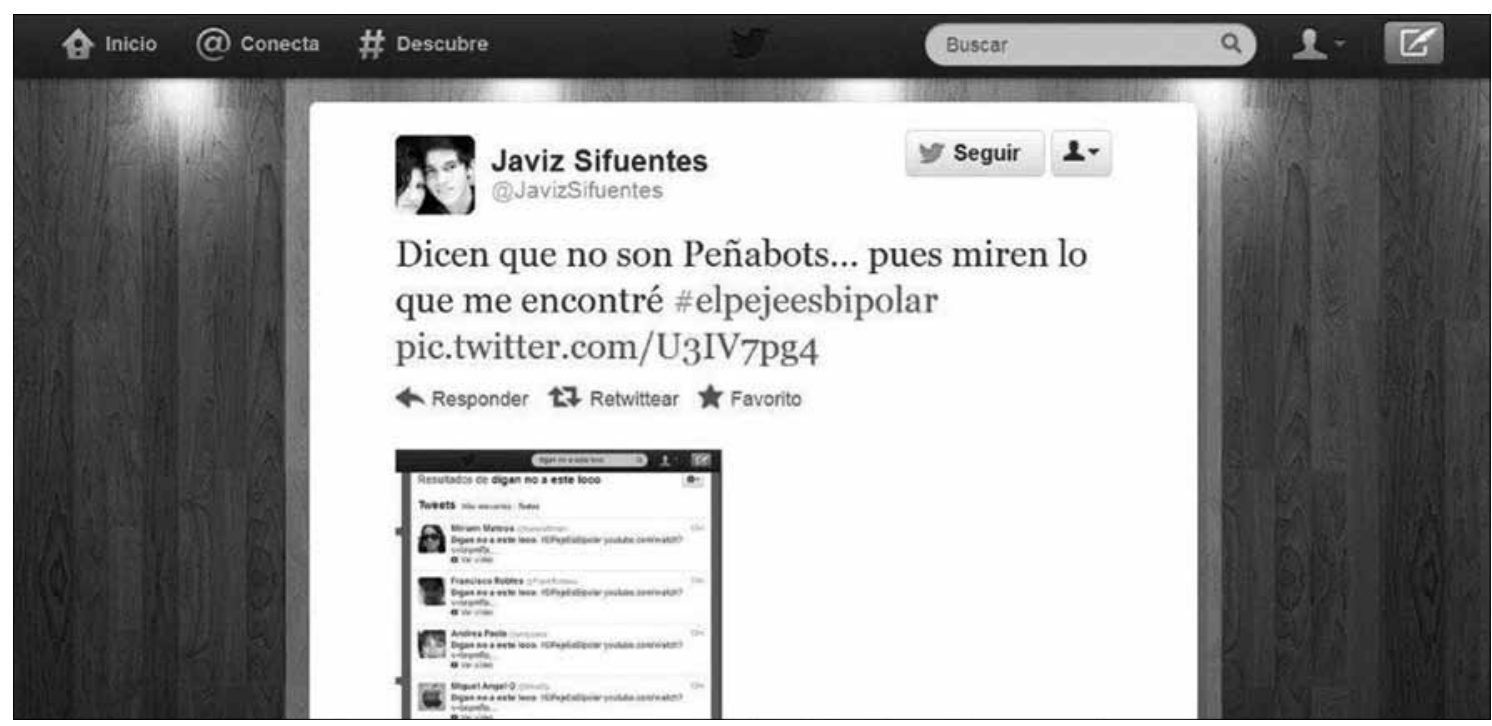

Peñabots: tuiteros contratados por el equipo de campaña de Enrique Peña Nieto.

Más que preguntarnos si las interacciones en esta esfera son auténticas, o si son consistentes las vidas virtuales y reales de las personas, el etnógrafo intenta

evaluar cómo se organiza y se vive una cultura en sus propios términos. La intención, por tanto, es dejar de lado la pregunta sobre la "verdadera" identidad, e incluso sobre si la "realidad" existe, y reorientar el foco empírico hacia cuestiones que tienen que ver con cómo, dónde y cuándo aparecen identidades y realidades en internet (Hine, 2000: 147).

En el contexto electoral de 2012, otro tema relacionado con el de la suplantación de identidad fue el de los usuarios "ficticios", que dio lugar a duros cuestionamientos sobre la forma en que algunos candidatos se esforzaron por crear una imagen de popularidad avalada por los cientos de miles de seguidores constatados en su perfil. Tal vez los usuarios ficticios más mencionados fueron los "peñabots", tuiteros "pirata" cuyas actividades sospechosas y atípicas - por ejemplo el retuiteo simultáneo del mismo texto por decenas de usuarios- comenzaron a ser denunciadas meses atrás. Su existencia quedó demostrada el 8 de mayo de 2012 en un video difundido con el trending topic \#elpriestaencrisis, en el que se mostraba cómo un coordinador de campaña instruía a un grupo de jóvenes para "ponerse a tuitear y revertir hashtags negativos”. Ahora, respecto de los destinatarios expresamente construidos en los tweets, se observó cómo la comunidad tuitera mantuvo un flujo constante de información al enviar mensajes de apoyo o crítica a los candidatos o personajes relacionados con la política, como vemos en los siguientes ejemplos dirigidos a los cuatro aspirantes a la presidencia:

\section{¡YoSoy132! @jramirorr}

¿QUE PASO @EPN? ¿NO TE ANIMAS IR AL DEBATE \#YoSoy132? SERA EN LA CDHDF, iY TIENEN BAÑOS!

\section{Carolina Gaytan@CarolinaGaytan1}

Ay Josefina mira que decir "Cuchi-cuchi” en vez de usar un término correcto como... "No mantener la relación sexual". Por ejemplo.

\section{Regina Solano @RegieSun}

${ }^{\star}$ Gabriel Quadri de la Torre, habla bien bonito pero no voy a votar por usted.

\section{Marco Antonio @MarcoAMLO}

@lopezobrador_mándeme un saludo por favor, yo lo admiro mucho, voy a votar por usted 


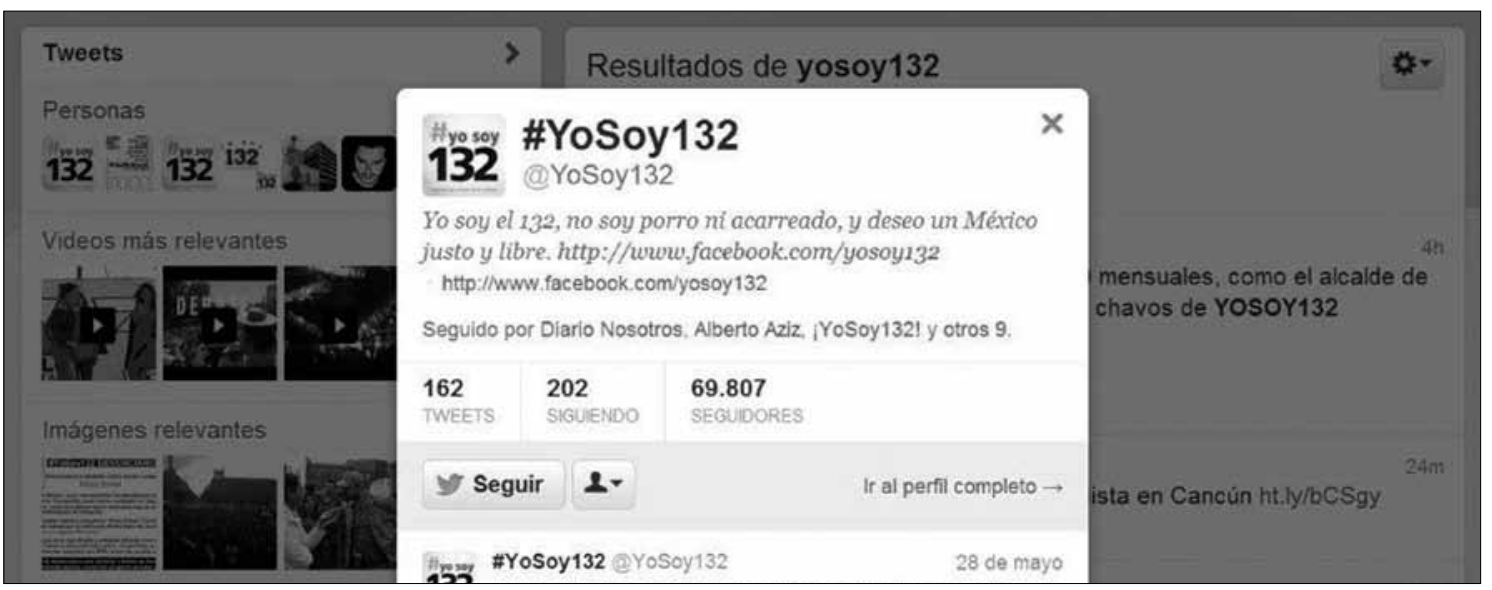

Perfil de Twitter del Movimiento \#YoSoy132.

En el marco de esta campaña, miles de tweets tenían como objetivo hacer llamados al IFE, a las televisoras, a las casas encuestadoras, a los simpatizantes, a los partidos, entre otros actores colectivos, para garantizar la legalidad y equidad de la contienda. El foro se aprovechó también para invitar a participar en las marchas a favor o en contra de los candidatos.

\section{¿QUÉ SE TUITEABA?}

Para explorar los miles de mensajes intercambiados en Twitter en el marco de la contienda electoral, conviene pensar tanto en la forma como en el contenido. Si nos centramos en la forma, encontramos que si bien Twitter impone la limitación aparente de 140 caracteres por mensaje, en realidad ésta ha quedado atrás. Podríamos afirmar, sin exagerar, parodiando a Arquímedes: "Dadme un tweet y recorreré el mundo", pues es común que estos 140 caracteres funcionen como un enlace - link - o hipertexto hacia otros mensajes, que a su vez conducen a otros mensajes en un recorrido interminable. En Lenguaje e internet, Crystal plantea que "la web es más ecléctica gráficamente que cualquier ámbito del lenguaje escrito en el mundo real" (Crystal, 2002: 227-228). El color, la imagen, el movimiento y otros mecanismos presentes en las páginas virtuales tienen como finalidad atraer la atención e impedir lecturas que sigan los cánones tradicionales. Frente a ello, dice Crystal, "el concepto del enlace de hipertexto es quizás el desafío más fuerte que ha de soportar la mirada inicial" (Crystal, 2002: 227). No obstante, limitar el texto inicial a 140 caracteres otorga a Twitter una eficacia política en contraste con otras redes sociales, como Facebook, ya que la cantidad ilimitada y la variedad de material circulante anquilosa el poder de convocatoria, pues es claro que no todos los internautas conocen la regla periodística de la síntesis informacional del primer párrafo. ${ }^{3}$ En este proceso electoral, Twitter se convirtió en un canal de comunicación extraordinario en el que los usuarios, en ocasiones después de un brevísimo entrenamiento digital, pudieron compartir artículos, fotos o twitpics, fotomontajes, canciones, poemas, chascarrillos, manifiestos, proclamas, denuncias, películas, clips de video y, desde luego, retweets - reenvío de otros tweets-, $\tan$ importantes para definir los trending topics - TT o tendencias-, en los que vale la pena detenernos.

\footnotetext{
${ }^{3}$ Debo éste y otros valiosos comentarios a Jorge Luis Méndez, uno de tantos tuiteros activos durante la campaña, a quien agradezco la lectura detallada de una primera versión de este escrito.
} 


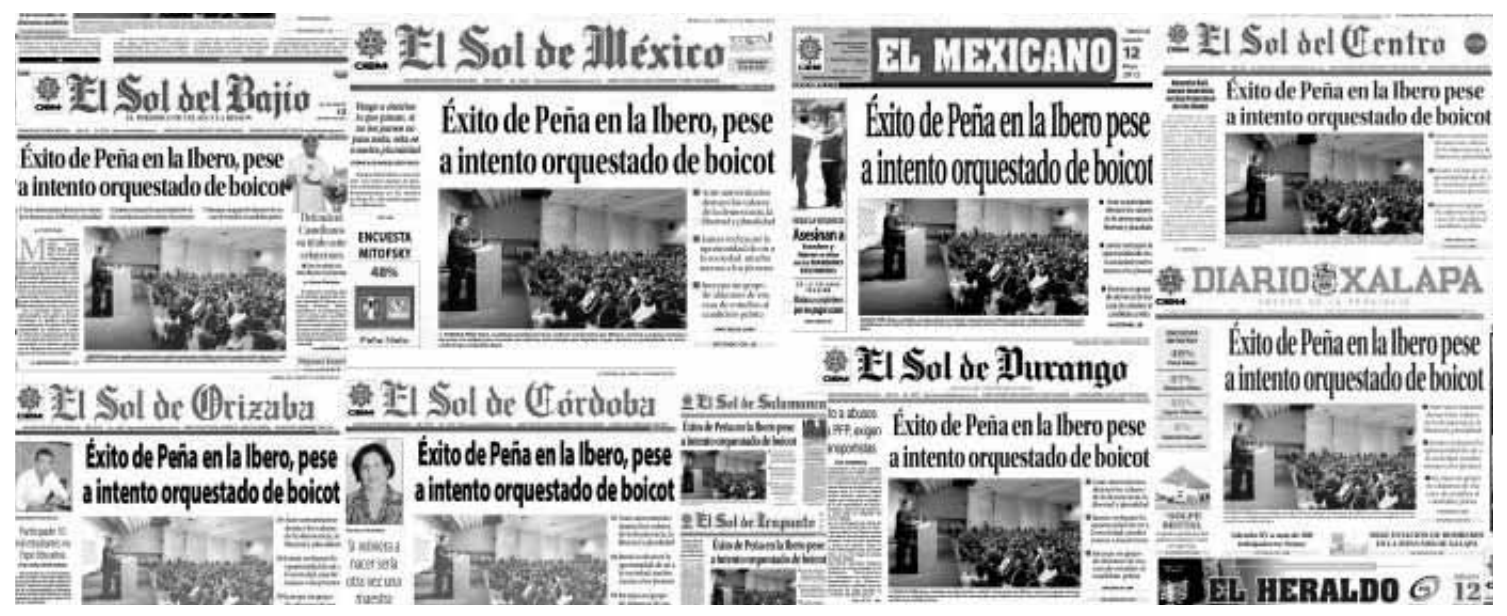

Titulares de periódicos de Organización Editorial Mexicana (OEM), 12 de mayo de 2012.

Según Wikipedia los trending topics, cuya traducción oficial al español es "tendencias", son las palabras o frases más repetidas en momentos específicos. Los 10 más relevantes se despliegan en la página de inicio de sesión en Twitter. El usuario puede escoger

el ámbito geográfico o personalizado de los тT. Tienen su origen en los hashtags (\#) o etiquetas, que sirven para organizar tuiteos sobre temas concretos. El potencial de estos trending topics resulta considerable, pues ofrecen información sobre los temas que ocupaban a la opinión pública mundial. Cabe señalar que a partir de junio de 2012 la página de inicio de Twitter ya no muestra una lista efectiva de los TT, sino listas personalizadas, o tailored trends, obtenidas por algoritmos que utilizan la ubicación del usuario y a quién sigue. En teoría, esta fabricación personalizada resultaría de más interés para el usuario; en el fondo, pareciera un mecanismo que resta potencial al libre flujo de informaciones y opiniones. Si bien en el periodo más álgido de la contienda electoral la lista de TT aún no era objeto de operaciones de personalización. De ahí la importancia de que numerosos TT se mantuvieran durante días en los primeros lugares a nivel mundial, lo que era un claro indicador de la efervescencia de la comunidad tuitera en México con motivo de los sucesos en torno a las elecciones. Hubo tendencias que marcaron episodios importantes o simplemente hilarantes y convirtieron a la comunidad tuitera en el termómetro de la contienda. Algunos casos célebres fueron las respuestas de EPN en el marco de la XV Feria Internacional del Libro de Guadalajara (FIL) -\#LibreriaPenaNieto fue TT mundial—, la reacción de la comunidad estudiantil de la Ibero durante la visita de Peña Nieto — que dio lugar al mencionado \#YoSoy132-, las marchas y otras formas de expresión pública - además de que Twitter fue un eficaz medio de convocatoria, \#MarchaAntiEPN se mantuvo durante varios días en los TT mundiales-, los debates organizados por el IFE y el del Movimiento \#YoSoy132, que tuvieron también amplia repercusión en esta red social y registraron interesantes fenómenos de recepción y retroalimentación crítica colectivas para llevar a cabo análisis en tiempo real. En relación con los mensajes que circulan en la red, Yus señala:

estos mensajes suelen ser excesivamente concisos en su codificación - a menudo suboracionales - y el lector ha de rellenar varios huecos informativos para convertir la esquemática forma lógica del mensaje -lo que el usuario teclea- en una interpretación finalmente relevante - lo que el usuario desea comunicar- (Yus, 2010: 162). 
Para comprender el sentido de muchos de estos TT, el nivel pragmático de la lengua resulta esencial. Sin elementos contextuales suficientes, ¿cómo saber a qué aluden TT tan crípticos como \#laganadorafuelaedecan, \#ladrocomoAdela o \#CuchiCuchiComoJVM, entre cientos más que hicieron historia en el primer semestre de 2012? Vistos en conjunto, los mensajes que circularon de manera profusa en Twitter daban testimonio de un sector de la sociedad ávido de participar en nuevas formas de acción democrática, por medio de un conjunto cada vez mayor de posibilidades tecnológicas que hacen posible la comunicación de inquietudes, insatisfacciones, aspiraciones, filias, fobias, emociones y un sinfín de añadidos relacionados con las elecciones en ciernes.

En esta exploración de los mensajes, vale la pena detenernos en el humor y el ingenio como elementos importantes, que no fueron ajenos al furor con el que miles de usuarios, principalmente jóvenes, se dieron a la tarea de tuitear. A fin de cuentas, miles de cabezas trinando simultáneamente trinaban mejor y más divertido que una. De los episodios que tenían al humorismo entre sus componentes destacados citemos algunos trending topics: \#LibreriaPenaNieto, que mostraba el azoro ante las pocas respuestas afortunadas del candidato priista en su visita a la FIL en diciembre de 2011; \#MeEscondoEnElBañoComoepn, para filtrar el rumor en torno a la salida furtiva del mismo candidato durante su visita a la Ibero; \#laganadorafuelaedecan, ante la insólita reacción del público televidente luego de la inexplicable aparición de una joven provocativamente ataviada al inicio del primer debate organizado por el IFE — que a la postre sería juzgado como una maniobra distractora de los temas sustanciales del debate-; \#CuchiCuchicomoJosefina, en respuesta al comentario de la candidata dirigido a un grupo de mujeres simpatizantes sobre sancionar a sus maridos: que si no votaban por ella se abstuvieran de darles "cuchi cuchi" durante un mes, declaración que despertó críticas enardecidas y sarcasmos al por mayor en la comunidad tuitera;
\#ladrocomoAdela, para referirse a la forma en que algunos conductores del programa Tercer Grado, entre ellos Adela Micha, se comportaron ante la visita de López Obrador al programa estelar de opinión de Televisa.

Por último, mencionamos el polémico tema de las encuestas - que se empeñaron en colocar a Enrique Peña Nieto como candidato puntero con una diferencia considerable respecto de López Obradorcuya metodología y con frecuencia sospechosos resultados fueron llevados a la palestra tuitera. Para confrontarlos se organizaron numerosos conteos en las redes sociales con la finalidad de medir el grado de aceptación en los candidatos, cuyos resultados favorecían por mayoría abrumadora a López Obrador. Si bien es cierto que el humor pudo ser un elemento de atracción para los tuiteros, sobre todo si consideramos que para buena parte de ellos la movilización política en redes sociales era una práctica relativamente novedosa, también lo es que la recurrencia a esta estrategia discursiva podría derivar en una banalización, dado que el aspecto crítico se erosiona paulatinamente y es reemplazado por una participación motivada casi de manera exclusiva por el afán lúdico. Tal vez una de las estrategias de las que se vale el poder sea tomar esta actitud estoica y complaciente, en la que se permiten ciertas libertades en apariencia críticas - como hacer mofa de personajes políticos-, sin que ello implique una toma de conciencia real de aquello que se impugna. En "El sujeto y el poder", Foucault advierte:

El poder sólo se ejerce sobre sujetos libres, y sólo en tanto ellos sean libres. Por esto entendemos sujetos individuales o colectivos que están enfrentados a un campo de posibilidades en el cual diversas formas de comportarse, diversas reacciones y comportamientos pueden ser realizados (Foucault, 1988: 16).

Bien vistas, actividades como reír o ironizar a propósito del poder y sus estructuras podrían figurar entre este campo de posibilidades, que, pese a todo, permiten a aquél salir indemne. 


\section{CONCLUSIONES}

Estas reflexiones son apenas un atisbo de lo que ocurrió en Twitter durante la contienda electoral. Queda un cúmulo de aspectos por evaluar para tener un panorama más completo del uso y las funciones desempeñadas por las redes sociales virtuales en este proceso. Por ejemplo, sería interesante analizar el conjunto de las redes como parte de un arsenal de nuevos dispositivos tecnológicos que permiten otras formas de comunicación, como Facebook -más popular que Twitter, al menos en términos cuantitativos- o Youtube, páginas que hacen viable que el intercambio de información y opiniones sea una práctica cada vez más horizontal, al alcance de un número creciente de usuarios y ajena a los monopolios mediáticos. Un estudio semejante llevaría a identificar más cabalmente los factores que han dotado a Twitter de mayor eficacia política respecto de Facebook, como que en el primero se distingue a los usuarios por "seguidos" y "seguidores", en tanto que en el segundo se propone un pacto en apariencia igualitario entre "amigos", denominación semántica que conlleva, de entrada, una actividad menos politizada. ¿Será posible que estas nuevas formas de expresión tengan un efecto verdaderamente revolucionario en el ámbito público, al extremo de hacer sentir la fuerza colectiva de los usuarios en torno a alguna causa común? El panorama dista de ser optimista a la luz de lo ocurrido en las elecciones de 2012 en las que, pese a los reclamos y demandas de numerosos colectivos, se comprobó que aún falta erradicar irregularidades y vicios del sistema electoral, como la compra de votos, que fue ampliamente documentada en redes sociales mas no logró revertir el fallo del IFE y posteriormente del Tribunal Electoral Federal (Trife). Las redes sociales virtuales demostraron su potencial, pero resultaron ser insuficientes todavía.

¿Cómo explicar este saldo pendiente del sistema político, sobre todo hacia la población joven? Quizá una de las respuestas esté en que las redes sociales coadyuvan a la movilización social, pero no reemplazan otras formas convencionales o, en todo caso, menos virtuales. Si reflexionamos en torno a la veloz gestación de \#YoSoy132, hay que admitir que las redes no fueron plenamente autosuficientes para determinar su creación. De inicio, se registró el interés de cada uno de los 131 estudiantes dispuestos a salir del anonimato e identificarse para revertir la información tendenciosa difundida principalmente en algunos medios impresos. La presencia de estos 131 jóvenes fue visualmente impactante y destruyó el estereotipo de la apatía política de los estudiantes inscritos en escuelas privadas, alimentó el espíritu colectivo que fraguaría en \#YoSoy132 y en su posterior movilización, que incluyó desde asambleas, movilizaciones y marchas hasta uno de los debates más democráticos de los que se tenga registro en la historia electoral reciente de México - al que no asistió el candidato del PRI- . Las redes sociales virtuales, en especial Twitter, fueron el canal, pero no el motor de esta historia. Concebidas como un elemento generador de la movilización social, su potencial es susceptible de aumentar y, sobre todo, de permanecer si se complementa con acciones más tangibles. También es pertinente recordar que los estudiosos de la comunicación han insistido hasta el hartazgo desde hace algunas décadas en que información no es comunicación. Las redes informan, pero no siempre comunican, y la diferencia entre un proceso y otro puede resultar abismal. Wolton señala:

Al mundializarse la información, el más menudo de
los acontecimientos se torna visible y, en apariencia,
más comprensible. Ahora bien, esto no significa que
exista un vínculo directo entre el incremento del
número de informaciones y la comprensión del mun-
do. Tal es el nuevo escenario del siglo que se inicia:
la información no crea comunicación (Wolton,
2003: 21-22).

Para comprender la relevancia de la dimensión cultural en la comunicación es preciso volver a las características de ésta. Ello implica tres dimensiones: 


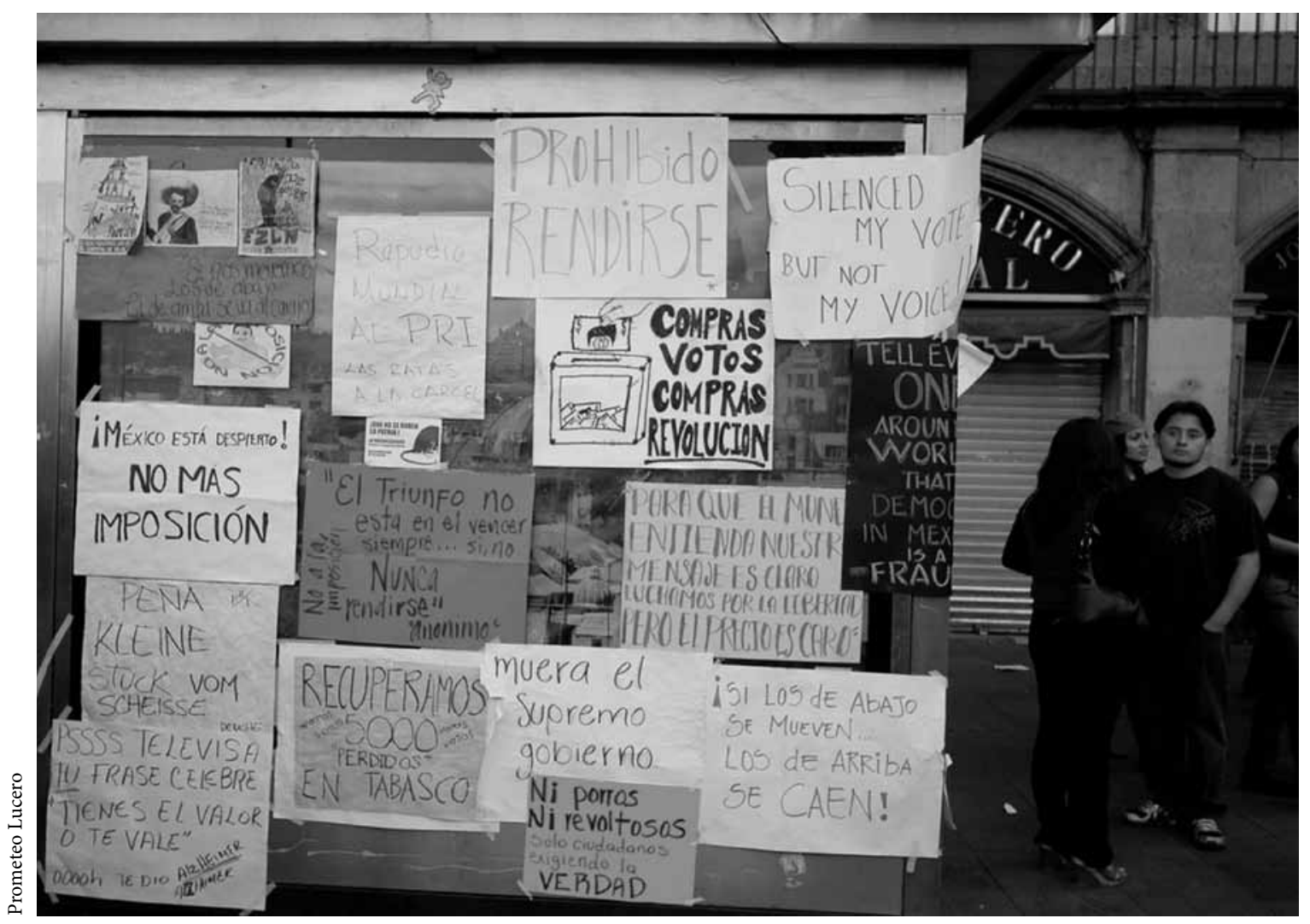

Marcha posterior a las elecciones e irrupción en la boda de Eugenio Derbez, ciudad de México, 7 de julio de 2012.

la técnica, la política y las condiciones socioculturales. Mientras que las dos primeras evolucionan con rapidez y en última instancia de modo paralelo, la tercera es la más compleja y de más lenta instalación. Los individuos cambian de herramientas más rápidamente de lo que cambian sus maneras de comunicarse. Para que se produzca una "revolución" en la comunicación es indispensable que haya una ruptura en los tres niveles. Hoy esta ruptura existe en los niveles técnico y económico, pero no aún en la tercera dimensión, que es también la más importante. Las técnicas y las redes no bastan para profundizar la intercomprensión: sucede incluso lo inverso. No hay que olvidar que, si bien las redes sociales y su amplia capacidad de convocatoria pueden generar movilizaciones de gran envergadura -como ocurrió en abril de 2011, cuando los ciudadanos de Egipto lograron derrocar al presidente Hosni Mubarak-, el poder no siempre resulta tan complaciente, tan fácil de derrocar o de dar marcha atrás.

En suma, la efervescencia del tema electoral en Twitter, algunos de cuyos trending topics determinaron la agenda mediática de la contienda política, revela el interés de una población, mayoritariamente joven, dispuesta a valerse de las redes sociales para hacer escuchar sus demandas por una verdadera democracia. Este potencial, no obstante, requiere ser fortalecido con otras formas de movilización que, a la postre, sean capaces de eliminar los lastres aún presentes en nuestro sistema político en general, que han impedido la consolidación de una democracia verdadera que se traduzca, a la larga, en una sociedad justa y equitativa. 


\section{BIBLIOGRAFÍA}

Aziz Nassif, Alberto, 2007, "El retorno del conflicto. Elecciones y polarización política en México", en Desacatos. Revista de Antropología Social, núm. 24, pp. 13-54.

Böckelmann, Franz, 1983, Formación y funciones sociales de la opinión pública, Gustavo Gili, Barcelona.

Bourdieu, Pierre, 1984, Cuestiones de sociología, Ediciones Istmo, Madrid.

Carr, Nicholas, 2011, Superficiales. ¿Qué está haciendo internet con nuestras mentes?, Taurus, México.

Crystal, David, 2002, El lenguaje e internet, Cambridge University Press, Madrid.

Foucault, Michel, 1988, "El sujeto y el poder", en Hubert L. Dreyfus y Paul Robinow, Michel Foucault: más allá del estructuralismo y la hermenéutica, Instituto de Investigaciones Sociales-Universidad Nacional Autónoma de México, México.

Gee, James Paul, 2002, An Introduction to Discourse Analysis: Theory and Method, Routledge, Gran Bretaña.

Hine, Christine, 2000, Etnografía virtual, Sage Publications, Barcelona.

Jakobson, Roman, 1981, Ensayos de lingüística general, Seix Barral, Barcelona.

Knapp, Mark, 1982, La comunicación no verbal. El cuerpo y el entorno, Paidós, Buenos Aires.

Kozinets, Robert V., 2010, Netnography, Doing Etnographic Research Online, Sage Publications, California.
Wolton, Dominique, 2003, La otra mundialización. Los desafíos de la cohabitación cultural global, Gedisa, Barcelona.

Yus, Francisco, 2010, Ciberpragmática. Nuevos usos del lenguaje en internet, Ariel, España.

\section{CIBERgRAFÍA}

go-gulf.com, 2012, "User Activity Comparison of Popular Social Networking Sites", infografía, 2 de mayo, en línea: $<$ http://www.go-gulf.com/blog/social-networkinguser >, consultado el 27 de octubre de 2011.

Islas, Octavio, 2012, "Estadísticas de Twitter en México", en Razón y Palabra, 17 de junio, en línea: <http:// www.razonypalabra.org.mx/espejo/ESPEJO_2011/ twitter.html>.

Jiménez Cano, Rosa, 2011, “Twitter cumple cinco años rodeado de polémica", en El País, 14 de marzo, en línea: <http://www.elpais.com/articulo/tecnologia/Twitter/ cumple/anos/rodeado/polemica/elpeputec/20110314 elpeputec_3/Tes $>$, consultado el 12 de agosto de 2011.

Reventós, Laia, 2008, "Míster Twitter", en El País, 17 de enero, en línea: <http://www.elpais.com/articulo/portada/Mister/Twitter/elpeputeccib/20080117elpcibp or_1/Tes $>$, consultada el 12 de agosto de 2011.

Twitter, Centro de Ayuda de Twitter, en línea: <support. twitter.com>.

Wikipedia, la enciclopedia libre, en línea: <es.wikipedia.org>. 